DOI: $10.17516 / 1999-494 X-0283$

УДК $53.09,627.67$

\title{
Detection of Sub-Surface Objects \\ Under Statistically Uneven Soil Surface \\ by Parametric Radar Method
}

\author{
Roman G. Shaydurov* \\ Siberian Federal University \\ Krasnoyarsk, Russian Federation
}

Received 30.08.2020, received in revised form 25.10.2020, accepted 27.11.2020

Abstract. The problem of sensing subsurface objects by the method of excitation of the earth's surface by Rayleigh seismic waves and obtaining information about the displacement of the ground above the target by radar method, taking into account the electromagnetic parameters of the underlying soil in the frequency range of $10 \mathrm{GHz}$, is considered. Recommendations are given on the optimal operating frequency of the radar in operating conditions on a statistically uneven ground surface.

Keywords: seismic waves, radar, parametrics, electromagnetic parameters, soil, surface.

Citation: Shaydurov R.G. Detection of sub-surface objects under statistically uneven soil surface by parametric radar method, J. Sib. Fed. Univ. Eng. \& Technol., 2020, 13(8), 1028-1033. DOI: 10.17516/1999-494X-0283

\section{Обнаружение подповерхностных объектов}

под статистически неровной поверхностью грунта

параметрическим радиолокационным методом

Р.Г. Шайдуров

Сибирский федеральный университет

Российская Федерачия, Красноярск

Аннотация. Рассмотрена задача зондирования подповерхностных объектов методом возбуждения поверхности земли сейсмическими волнами Рэлея и получения информации о смещении грунта над объектом поиска радиолокационным способом с учетом электромагнитных параметров подстилающего грунта в диапазоне частот 10 ГГц. Даны

(C) Siberian Federal University. All rights reserved

This work is licensed under a Creative Commons Attribution-Non Commercial 4.0 International License (CC BY-NC 4.0).

* Corresponding author E-mail address: rshaydurov@sfu-kras.ru 
рекомендации об оптимальной рабочей частоте радиолокатора в условиях работы на статистически неровной поверхности грунта.

Ключевые слова: сейсмические волны, радиолокация, параметрика, электромагнитные параметры, грунт, поверхность.

Цитирование: Шайдуров, Р.Г. Обнаружение подповерхностных объектов под статистически неровной поверхностью грунта параметрическим радиолокационным методом / Р.Г. Шайдуров // Журн. Сиб. федер. ун-та. Техника и технологии, 2020. 13(8). C. 1028-1033. DOI: 10.17516/1999-494X-0283

\section{Введение}

В ряде научных работ [1-3] рассматривается перспективный параметрический метод поиска подповерхностных объектов. Сущность метода заключается в возбуждении в объектах поиска собственных механических колебаний с помощью сейсмического излучателя поверхностных акустических волн Рэлея. Реакция объектов поиска на внешнее воздействие дистанционно переносится на радиолокационный сигнал, создаваемый радиолокатором СВЧ-диапазона.

При этом в отраженном радиолокационном сигнале появляется дополнительный информационный признак объекта - параметрическая модуляция его фазы механическими колебаниями оболочки объекта.

Целью данной статьи является теоретическая оценка основных энергетических параметров метода с учетом неоднородности поверхности среды зондирования.

\section{Постановка задачи}

На рис. 1 изображена схема возбуждения объекта поиска сейсмическим излучателем волн Рэлея 1 и сканирование подповерхностного пространства радиолокатором (РЛС), включающим передатчик 2, с антенной 3 и приемником 4 с приемной антенной 5 . Под поверхностью грунта 6 на глубине $h_{0}$ располагается объект поиска в виде металлического шара радиусом $R_{0}$. Вдоль поверхности распространяется сейсмическая волна Рэлея 8, а передатчиком РЛС облучается

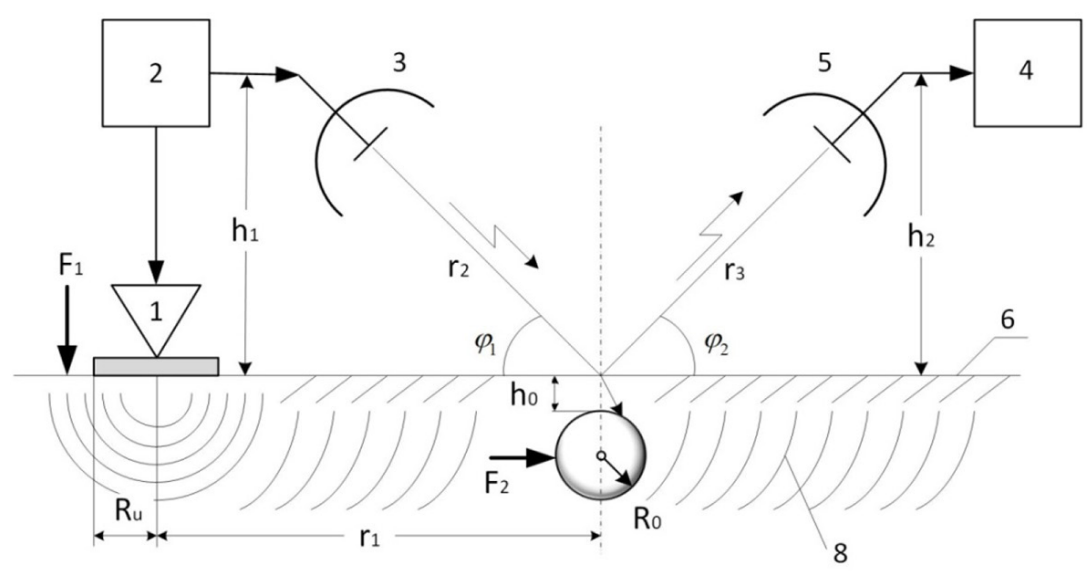

Рис. 1. Схема реализации радиолокационного параметрического способа обнаружения подповерхностных объектов

Fig. 1. Implementation diagram of the radar parametric method subsurface object detection 
пространство в точке положения объекта поиска. Отраженный в этой точке радиосигнал передатчика РЛС принимается приемником 4. Электромагнитные характеристики почвы определяются параметрами электропроводности $\sigma_{1}$, диэлектрической проницаемостью $\varepsilon$, магнитной проницаемостью $\mu$, а неровность границы раздела земля-воздух - эффективной поверхностью рассеивания $\sigma_{\Gamma}$. Передающая и приемная антенны имеют горизонтальную поляризацию с ко-

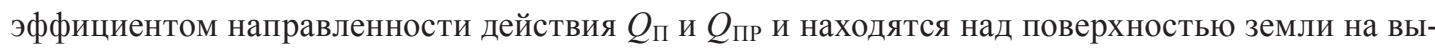
сотах $h_{1}$ и $h_{2}$ соответственно.

Под действием сейсмических ударов с силой $F_{1}$ давление волны Рэлея на боковую поверхность объекта в виде шара радиусом $R_{O}$ можно оценить через соотношение [1]

$$
F_{2}=\frac{F_{1}}{\pi R_{u}^{2}} \cdot \frac{R_{u} S}{r_{1}} e^{-\alpha r_{1}},
$$

где $\alpha=10^{-3} \frac{1}{M}-$ коэффициент поглощения акустической волны в грунте, $R_{u}$ - радиус излучающей плиты сейсмического источника. При этом смещение корпуса шара по направлению $x$ составит:

$$
\Delta x=\frac{F_{2} \tau^{2}}{m}
$$

где $S=\pi R_{O}^{2}-$ площадь бокового сечения шара (полусферы), $m=\frac{4}{3} \pi R_{O}^{3} \rho_{O}-$ масса шара, $\rho_{O}-$ его плотность.

Поверхность грунта облучается передатчиком 2 радиолокатора под углом скольжения $\varphi_{1}$ с расстояния $r_{2}$. Радиолокационный луч имеет мощность передатчика $P_{\text {П и коэффициент }}$ направленности действия (КНД) передающей антенны $Q_{\Pi}$. Отраженный сигнал от шероховатой поверхности в точке положения объекта на входе приемной антенны 5 оценивается как

$$
P_{I I P 1}=\frac{P_{\Pi} Q_{\Pi} \sigma_{0} A_{\Pi P} \eta}{4 \pi r_{2}^{2} \cdot 4 \pi r_{3}^{2}},
$$

где $\sigma_{0}-$ эффективная поверхность рассеивания ровной поверхности грунта; $A_{\text {Пр }}-$ эффективная площадь приемной антенны; $\eta$ - коэффициент отражения от шероховатой поверхности. Согласно [4] $\eta$ составит:

$$
\eta=\frac{\sigma_{\Gamma}}{\sigma_{0}}
$$

где $\sigma_{\Gamma}=4 S \rho$ - ЭПР шероховатой поверхности; $\rho$ - коэффициент отражения от шероховатой поверхности для горизонтальной поляризации РЛС (считаем $\rho=1$ ).

Сигнал на входе приемной антенны в этом случае будет иметь флуктуации фазы со среднеквадратичным значением:

$$
\Delta \varphi_{\Gamma}=2 \pi \frac{H}{\lambda}
$$

где $H$ - среднеквадратичная высота неровностей грунта; $\lambda$ - длина волны передатчика РЛС.

При учете вибрации от источника сейсмических волн в отраженном сигнале появляется дополнительный сдвиг фаз величиной 


$$
\Delta \varphi_{O}=2 \pi \frac{\Delta x}{\lambda}
$$

а мощность отраженного сигнала на входе приемной антенны от объекта составит:

$$
P_{I I P 2}=\frac{P_{I I} Q_{I I} \sigma_{I I} A_{I I P}}{(4 \pi)^{2} r_{2}^{2} r_{3}^{2}} e^{-2 \alpha_{\Gamma} h_{0}}
$$

где $\alpha_{\Gamma}=\sqrt{\frac{\omega \mu \sigma_{1}}{2}}-$ коэффициент затухания электромагнитной волны в грунте; $\sigma_{1}-$ электропроводность грунта; $\mu=4 \pi \cdot 10^{-7} \frac{\Gamma_{H}}{M}-$ магнитная проницаемость грунта; $\omega=2 \pi \mathrm{f}-$ рабочая частота передатчика; $\sigma_{\Psi}=2 \pi R_{0}^{2}-$ ЭПР объекта.

При вибрации корпуса объекта поиска под воздействием сейсмической волны ЭПР шара будет испытывать модуляцию с амплитудой

$$
\Delta \sigma_{\amalg}=\sigma_{\amalg}\left(\frac{\Delta x}{\lambda}\right)^{2} .
$$

Отношение мощностей отраженного полезного сигнала от объекта поиска в виде шара и неровной поверхности составит:

$$
q_{X}=\frac{P_{\Pi P 2}}{P_{\Pi P 1}}=\frac{\sigma_{0}+\sigma_{\amalg} \frac{\Delta x^{2}}{\lambda^{2}}}{\sigma_{0} \eta} e^{-2 \alpha_{\Gamma} h_{0}} .
$$

Модуляцией ЭПР шара при воздействии сейсмической волны $\Delta \sigma_{ш}$ из-за малой величины в расчетах отношения мощностей можно пренебречь. Тогда при отсутствии вибрации корпуса объекта, когда $\Delta x=0$, отношение сигнал/помеха составит:

$$
q_{O}=\frac{\sigma_{I I}}{\sigma_{o} \eta} .
$$

Величину ЭПР ровной поверхности грунта примем равной ЭПР поверхности шара $\sigma_{\mathrm{m}}=\sigma_{0}=3,14 \cdot 10^{-2} \mathcal{M}^{2}$, тогда получим $q_{0}<1$, в этих условиях мы будем наблюдать большой уровень ложных тревог. В классическом варианте для снижения этих помех используется дифференциальный метод с приемом сигнала на две парно расположенные антенны так, что на поверхности грунта будет наблюдаться два смежных отраженных пятна с расстоянием между их центрами $R_{\amalg}$. Это позволяет частично компенсировать сигналы, отраженные от грунта. В случае параметрического метода при использовании двух приемных антенн сигналы с их выходов $U_{C 1}, U_{C 2}$ подаются непосредственно на смеситель, который в результате их перемножения выделяет с помощью фильтра нижних частот полезную составляющую сигнала в виде

$$
\Delta U_{C}=U_{C 1} \operatorname{Sin}\left(\omega t+\varphi_{1}\right) \otimes U_{C 2} \operatorname{Sin}\left(\omega t+\varphi_{1}+\Delta \varphi\right)=U_{\Delta} \frac{\Delta x}{x},
$$

где $U_{\Delta}=\sqrt{U_{C 1} U_{C 2}}$.

Из (2) найдем для примера численные значения смещения корпуса объекта $\Delta x$ при заданной силе удара сейсмического источника:

$$
\Delta x=\frac{F_{1} e^{-\alpha_{1} r}}{R_{u} r_{1}} \cdot \frac{R_{0}^{2} \tau^{2}}{M},
$$


где $M=m_{\mathrm{O}}+m_{\Gamma}-$ масса сдвигаемой среды, включающая массу самого объекта $m_{\mathrm{O}}$ и так называемую массу присоединенного грунта $m_{\Gamma}$.

Расчет проведем для $M=m_{\mathrm{O}}+m_{\Gamma}=10$ кг; $F_{1}=10^{3}$ кГ; $\tau=5 \cdot 10^{-3} \mathrm{c} ; \lambda_{1}=10^{-3} \frac{1}{M} ; r_{1}=10 \mathrm{м}$. Тогда $\Delta x=25 \cdot 10^{-6}$ м.

Считая, что фазовым детектированием можно получить точность порядка $1^{\circ}$, из соотношения (6) получим, что смещение корпуса объекта при воздействии сейсмической волны должно составить величину не менее

$$
\Delta x \geq \frac{\Delta \varphi \lambda}{2 \pi} \approx 10^{-4} \mathcal{M}
$$

Через (1) можно определить необходимую силу удара сейсмического источника:

$$
F_{1}=\frac{\Delta x R_{u} r_{1} M}{R_{O}^{2} \tau^{2} e^{-2 \alpha_{1} r_{1}}}
$$

Поскольку оценка по (1) дает силу удара (около 4 т), то с целью увеличения отношения сигнал/шум необходимо использовать накопление сигнала за счет повтора сейсмических импульсов. Например, при четырех импульсах за секунду появляется возможность повысить отношение сигнал/шум по мощности в 4 раза. Это позволит снизить силу удара до 2 т. Данный способ эффективен для снижения влияния шероховатости поверхности грунта при их размерах, составляющих менее длины волны РЛС.

С другой стороны, современные методы цифровой обработки сигналов позволяют повысить чувствительность метода при оценке фазового сдвига до $0,1^{\circ}$, что дает дополнительный потенциал для снижения силы сейсмического воздействия и конструирования более компактных и менее энергоемких источников. Кроме этих мер принципиальное увеличение чувствительности метода и снижение мощности сейсмических источников дает увеличение рабочей частоты РЛС вплоть до миллиметрового диапазона (порядка 100 ГГц) и оптического с длиной волны в диапазоне инфракрасного спектра. Поскольку расстояние от передатчика до цели относительно мало, то увеличение частоты не приведет к сложностям эксплуатации в условиях повышенной влажности воздуха и естественных помех.

\section{Заключение}

1. Шероховатость поверхности почвы с размерами меньше длины волны РЛС может эффективно подавляться повышением частоты генерации сейсмических ударов при использовании параметрического метода.

2. Повышение чувствительности этого метода и снижение силы ударов сейсмического источника наиболее целесообразны путем снижения рабочей длины волны передатчика и использования, в том числе методов дифференциального приема сигналов. 


\section{Благодарности / Acknowledgments}

Работа выполнена при финансовой поддержке РФФИ в рамках научного проекта № 18-07$00180 \mathrm{~A}$.

This work was supported by the Russian Foundation for Basic Research within the framework of a research project No. 18-07-00180 A.

\section{Список литературы / References}

[1] Шайдуров Р.Г. Параметрическая роботизированная система поиска мин на основе взаимодействия электромагнитных и акустических волн. Журнал Сибирского федерального университета. Техника и технологии, 2018, 11(7), 755-763 [Shaydurov R.G. Parametric robotic mine search system based on the interaction of electromagnetic and acoustic waves. Journal of the Siberian Federal University. Engineering \& Technologies, 2018, 11 (7), 755-763 (in Russian)]

[2] Donskoy D.M. Nonlinear vibro-acoustic technique for landmine detection. Proc. SPIE' 12th, Conference on Detection and Remediation Technologies for Mines and Minelike Targets IV, ed. By Dubey A.C. et al. 1998, pp. 211-217.

[3] Robert W. Haupt and Kenneth D. Rolt. Standoff Acoustic Laser Technique to Locate Buried Land Mines, Lincoln laboratory journal, 2005, 1(15), 3-23.

[4] Радиотехнические системы. Под ред. Ю.М. Казаринова. М.: Советское радио, 1968. 496 c. [Radio engineering systems. Editor Yu.M. Kazarinov. M., Soviet radio, 1968, 496 p. (in Russian)] 